\title{
Orexin B/hypocretin 2 increases glutamatergic transmission to ventral tegmental area neurons
}

\author{
S. L. Borgland, ${ }^{1, \star}$ E. Storm ${ }^{1}$ and A. Bonci ${ }^{1,2}$ \\ ${ }^{1}$ Ernest Gallo Clinic and Research Center, Department of Neurology, University of California, San Francisco, CA, USA \\ ${ }^{2}$ Wheeler Center for the Neurobiology of Addiction, University of California, San Francisco, San Francisco, CA, USA
}

Keywords: AMPA, dopamine, motivation, NMDA, orexin B, VTA

\begin{abstract}
The orexins (hypocretins) play a crucial role in arousal, feeding and reward. Highly relevant to these functions, orexin-containing neurons from the lateral hypothalamus project densely to the ventral tegmental area (VTA), which is the origin of dopamine projections implicated in motivation and reward. Orexin A/hypocretin 1 (oxA/hcrt-1) can enable long-term changes associated with drugs of abuse; however, the effects of orexin B/hypocretin 2 (oxB/hcrt-2) on excitatory synaptic transmission in the VTA are unknown. We used whole-cell patch-clamp electrophysiology in rat horizontal midbrain slices to examine the effects of oxB/hcrt-2 on excitatory synaptic transmission. We observed that oxB/hcrt-2 has distinct effects from oxA/hcrt-1 in the VTA. oxB/Hcrt-2 (100 nM) increased presynaptic glutamate release in addition to a postsynaptic potentiation of NMDA receptors (NMDARs). The oxB/hcrt-2-mediated postsynaptic potentiation of NMDARs was mediated via activation of orexin/hypocretin 2 (OX2/Hcrt-2) receptors and protein kinase C $(\mathrm{PKC})$. Furthermore, the increase in transmitter release probability was also PKC-dependent, but not through activation of orexin/hypocretin $1(\mathrm{OX} 1 / \mathrm{Hcrt}-1)$ or OX2/Hcrt-2 receptors. Finally, oxB/hcrt-2 or the selective OX2/Hcrt-2 receptor agonist $\mathrm{ala}^{11}$-D-leu ${ }^{15}$-orexin B, significantly reduced spike-timing-induced long-term potentiation. Taken together, these results support a dual role for oxB/hcrt-2 in mediating enhanced glutamatergic transmission in the VTA, and suggest that oxA/hcrt-1 and oxB/hcrt-2 exert different functional roles in modulating the enhancement of the motivational components of arousal and feeding.
\end{abstract}

\section{Introduction}

Ventral tegmental area (VTA) neurons are critically involved in reward, arousal and appetitive behaviors (Grace et al., 1998; Carlezon $\&$ Nestler, 2002; Berridge, 2007). VTA neurons are the main source of dopamine for the ventral striatum and prefrontal cortex, critical forebrain regions that mediate incentive learning and reinforcement mechanisms associated with positive reward (Berridge, 2007). Excitatory synaptic transmission onto VTA dopamine neurons increases locomotor activity (Dunn et al., 2005) and is an important locus of neural plasticity induced by psychostimulant administration (Fitzgerald et al., 1996; Ungless et al., 2001; Saal et al., 2003; Borgland et al., 2004).

Two neuropeptides, orexin A/hypocretin 1 (oxA/hcrt-1) and orexin $\mathrm{B} /$ hypocretin 2 (oxB/hcrt-2), have also been implicated in arousal, feeding and reward (DeLecea et al., 1998; Sakurai et al., 1998; Georgescu et al., 2003; Yamanaka et al., 2003; Boutrel et al., 2005; Harris et al., 2005; Borgland et al., 2006; Narita et al., 2006). These peptides are synthesized in neurons exclusively localized to the lateral

Correspondence: Dr S. L Borgland, *present address as below.

E-mail: borgland@interchange.ubc.ca

*Present address: Department of Anesthesiology, Pharmacology \& Therapeutics, University of British Columbia, Rm 212, 2176 Health Sciences Mall, Vancouver, BC, Canada V6T 1 Z3.

Received 12 November 2007, revised 11 June 2008, accepted 4 July 2008 hypothalamic area and project widely through the brain (Peyron et al., 1998; Sakurai et al., 1998). The VTA receives an input from orexincontaining neurons (Fadel \& Deutch, 2002; Baldo et al., 2003) composed of both synaptic terminals and en-passant fibers (BalcitaPedicino \& Sesack, 2007), and this input is a critical element in motivation and reward circuits activated by drugs of misuse (Wise, 1996; Harris \& Aston-Jones, 2006).

The actions of orexins are mediated by two known G-proteincoupled receptors termed orexin/hypocretin receptor 1 (OX1/hcrt-1) and orexin/hypocretin receptor 2 (OX2/hcrt-2; Sakurai et al., 1998). oxA/hcrt-1 has high affinity for both receptors whereas oxB/hcrt-2 has much higher affinity for OX2/hcrt-2 than OX1/hcrt-1 receptors (Sakurai et al., 1998). Both receptors are expressed on VTA dopamine and GABAergic neurons (Korotkova et al., 2003; Narita et al., 2006). Furthermore, both oxA/hcrt- 1 and $\mathrm{oxB} / \mathrm{hcrt}-2$ increased the firing rate of VTA dopamine or GABAergic neurons in vitro (Korotkova et al., 2003). oxA/hcrt-1 or oxB/hcrt- 2 also activated phosopholipase $\mathrm{C}$ - and protein kinase $\mathrm{C}$ (PKC)-mediated $\mathrm{Ca}^{2}$ signaling in dopamine neurons (Uramura et al., 2001; Narita et al., 2007), and we have previously reported that oxA/hcrt-1 potentiates NMDA receptor (NMDAR)mediated synaptic transmission in the VTA via a PKC-dependent translocation of the receptors to the synapse (Borgland et al., 2006).

A role for oxA/hcrt-1 and OX1/Hcrt-1 receptors in mediating some of the effects of drugs of abuse has recently been defined (Boutrel et al., 2005; Harris et al., 2005; Borgland et al., 2006; 
Lawrence et al., 2006; Narita et al., 2006, 2007). OX2/Hcrt-2 receptors have been thought to play a primary role in mediating wakefulness and arousal, as OX2/Hcrt-2 knockout mice experience narcolepsy (Chemelli et al., 1999; Akanmu \& Honda, 2005). Although intracerebroventricular administration of oxB/hcrt-2 has been shown to increase locomotor activity (Narita et al., 2006) and intra-VTA administration increases preference for morphine (Narita et al., 2007), it is unknown if these effects of oxB/hcrt-2 are mediated through activation of $\mathrm{OX} 2 / \mathrm{Hcrt}-2$ receptors. Enhancement of glutamatergic synaptic transmission in VTA dopamine neurons has been proposed to underlie some of the long-term changes associated with chronic drug use (Borgland et al., 2004; Kauer, 2004; Wolf et al., 2004), but the effects of oxB/hcrt-2, which is presumably co-released with oxA/hcrt-1, on synaptic plasticity in the VTA are unknown. Therefore, using whole-cell patch clamp, we examined the role of $\mathrm{oxB} /$ hcrt-2 on excitatory synaptic transmission in VTA neurons.

\section{Methods and materials}

\section{Electrophysiology}

All electrophysiological recordings were performed in male SpragueDawley rats ranging from postnatal day (P)21 to P30. All experiments were performed according to NIH and UCSF guidelines on animal use. Briefly, rats were anesthetized with halothane and decapitated. Horizontal sections of the VTA $(230 \mu \mathrm{M})$ were prepared with a vibratome (Leica, Nussloch, Germany). Slices were placed in a holding chamber and allowed to recover for at least $1 \mathrm{~h}$ before being placed in the recording chamber and superfused with bicarbonatebuffered solution (artificial cerebrospinal fluid; aCSF) saturated with 95\% $\mathrm{O}_{2} / 5 \% \mathrm{CO}_{2}$ and containing (in $\mathrm{mM}$ ): $119 \mathrm{NaCl}, 1.6 \mathrm{KCl}, 1.0$ $\mathrm{NaH}_{2} \mathrm{PO}_{4}, 1.3 \mathrm{MgCl}_{2}, 2.5 \mathrm{CaCl}_{2}, 26.2 \mathrm{NaHCO}_{3}$ and 11 glucose (at $\left.32-34{ }^{\circ} \mathrm{C}\right)$. For all experiments, picrotoxin $(100 \mu \mathrm{M})$ was added to block $\mathrm{GABA}_{\mathrm{A}}$ receptor-mediated inhibitory postsynaptic currents. Cells were visualized using infrared differential interference contrast video microscopy. Whole-cell voltage-clamp recordings were made using an Axopatch 1D amplifier (Axon Instruments, Union City, CA, USA). Electrodes (3-4.0 M 2 ) contained (in mM): 120 cesium methansulfonate, 20 HEPES, 0.4 EGTA, $2.8 \mathrm{NaCl}, 5$ TEA-Cl, 2.5 MgATP, and 0.25 NaGTP, pH 7.2-7.3 (270-285 mOsm). Series resistance (10-40 M $\Omega$ ) and input resistance were monitored on-line with a 4-mV depolarizing step $(50 \mathrm{~ms})$ given just after every afferent stimulus. Neurons were visualized with an upright microscope using infrared differential interference contrast illumination. Putative dopaminergic VTA neurons were identified by the presence of a large hyperpolarization-activated, cyclic nucleotide-regulated cation $\left(I_{\mathrm{h}}\right)$ current (Grace \& Onn, 1989; Lacey et al., 1990; Johnson \& North, 1992; Cameron et al., 1997; Ford et al., 2006) and were selected from the population of neurons surrounding the medial side of the medial terminal nucleus of the accessory optic tract, a region with a substantial population of $I_{\mathrm{h}}$-positive dopamine neurons (Margolis et al., 2007). We recognize that $I_{\mathrm{h}}$ is present in both principal and tertiary VTA neurons and that the presence of $I_{\mathrm{h}}$ does not unequivocally identify dopamine neurons from other non-GABAergic neurons in midbrain slices (Margolis et al., 2007). In some cases, neurons were identified by biocytin $(0.2 \%)$ filling and post hoc staining for tyrosine hydroxylase (TH; see below). A bipolar stimulating electrode was placed $100-300 \mu \mathrm{m}$ rostral to the recording electrode and was used to stimulate excitatory afferents at $0.1 \mathrm{~Hz}$. Neurons were voltageclamped at -70 and $+40 \mathrm{mV}$ to record AMPAR- and NMDARmediated evoked excitatory post-synaptic currents (eEPSCs). eEPSCs were filtered at $2 \mathrm{kHz}$, digitized at $5-10 \mathrm{kHz}$ and collected on-line using Igor Pro software (Wavemetrics, Lake Oswego, OR, USA). Paired-pulse ratio was elicited by evoking two EPSCs with an interval of $50 \mathrm{~ms}$. Miniature EPSCs (mEPSCs) in the presence tetrodotoxin $(500 \mathrm{nM})$ to block action potentials driven by spontaneous events were recorded using PCLAMP 8 software (Axon Instruments). For these experiments, mEPSCs were recorded before and $10 \mathrm{~min}$ after a 5 -min application of oxB/hcrt-2 (100 nM, $5 \mathrm{~min})$. We did not observe a time effect of mEPSC event size or frequency in control neurons (data not shown). mEPSCs were selected based on their amplitude $(>8 \mathrm{pA})$, decay time $(<3 \mathrm{~ms})$ and rise time $(<1 \mathrm{~ms})$ using the MiniANALYSIS program (SYNAPTOSOFT). Average noise levels were $2 \pm 0.2 \mathrm{pA}$.

Amplitudes of currents evoked by bath application of NMDA $(20 \mu \mathrm{M}, 30 \mathrm{~s})$ before and $10 \mathrm{~min}$ after a 5 -min oxB/hcrt-2 application were determined by subtracting the baseline (average of 2 min before NMDA application) from the NMDA-induced current.

\section{Spike timing-dependent LTP}

Neurons were patch clamped in current-clamp mode with electrodes containing: $0.95 \% \mathrm{KOH}(\mathrm{v} / \mathrm{v}), 0.76 \%$ methanesulfonic acid $(\mathrm{v} / \mathrm{v})$, $0.18 \%$ hydrochloric acid (v/v), $20 \mathrm{mM}$ HEPES, $0.2 \mathrm{mM}$ EGTA, $2.8 \mathrm{mM} \mathrm{NaCl}, 2.5 \mathrm{mg} / \mathrm{mL} \mathrm{MgATP}, 0.25 \mathrm{mg} / \mathrm{mL}$ GTP and $0.2 \%$ biocytin, $\mathrm{pH} 7.2-7.4$ (275-285 mOsm). To induce LTP, the spiketiming protocol consisted of 20 bursts of excitatory post-synaptic potential (EPSP)-spike pairs, with each burst consisting of five paired stimuli delivered at $100-\mathrm{ms}$ intervals $(10 \mathrm{~Hz})$ and an inter-burst interval of $5 \mathrm{~s}$. The postsynaptic spikes were evoked by injection of depolarizing current pulses (1-2 nA, 2-3 ms), with the onset of EPSPs preceding the peak of the postsynaptic spikes by $5 \mathrm{~ms}$ (Liu et al., 2005). eEPSPs were sampled at $0.1 \mathrm{~Hz}$ before and after LTP induction. For quantifying LTP, we averaged the mean of 30 sweeps 5 min before drug treatment (baseline) and averaged 30 sweeps 25 min after the pairing protocol (peak LTP).

\section{TH immunocytochemistry}

Brain slices from patch-clamp recording were fixed overnight in cold $4 \%$ paraformaldehyde, rinsed in PBS, blocked in $10 \%$ normal donkey serum, incubated with monoclonal mouse anti-TH antibody $(1: 3000$; Sigma) and Avidin-Texas Red (1 : 500; Vector Labs) for $48 \mathrm{~h}$ at $4{ }^{\circ} \mathrm{C}$. Secondary donkey anti-mouse Alexa 488 antibody (1:500) was applied overnight. Slices were mounted using Vectashield (Vector Labs).

\section{Data analysis}

Mean potentiation of NMDAR or AMPAR currents by oxB/hcrt-2 represents the mean EPSCs over a period of $2 \mathrm{~min}, 25 \mathrm{~min}$ after application of $\mathrm{oxB} / \mathrm{hcrt}-2$. All values are expressed as mean $\pm \mathrm{SEM}$. Statistical significance was assessed using two-tailed Student's $t$-tests or a one-way ANOVA for multiple group comparisons. A Bonferroni post-hoc test following an ANOVA was used to test for significant differences between multiple groups.

\section{Results \\ oxB/Hcrt-2 enhances excitatory synaptic transmission in VTA neurons}

eEPSCs were recorded from VTA neurons in rat midbrain slices. NMDAR EPSCs were evoked while holding neurons in voltage clamp at $+40 \mathrm{mV}$. Measurements were taken $20 \mathrm{~ms}$ after the stimulus 

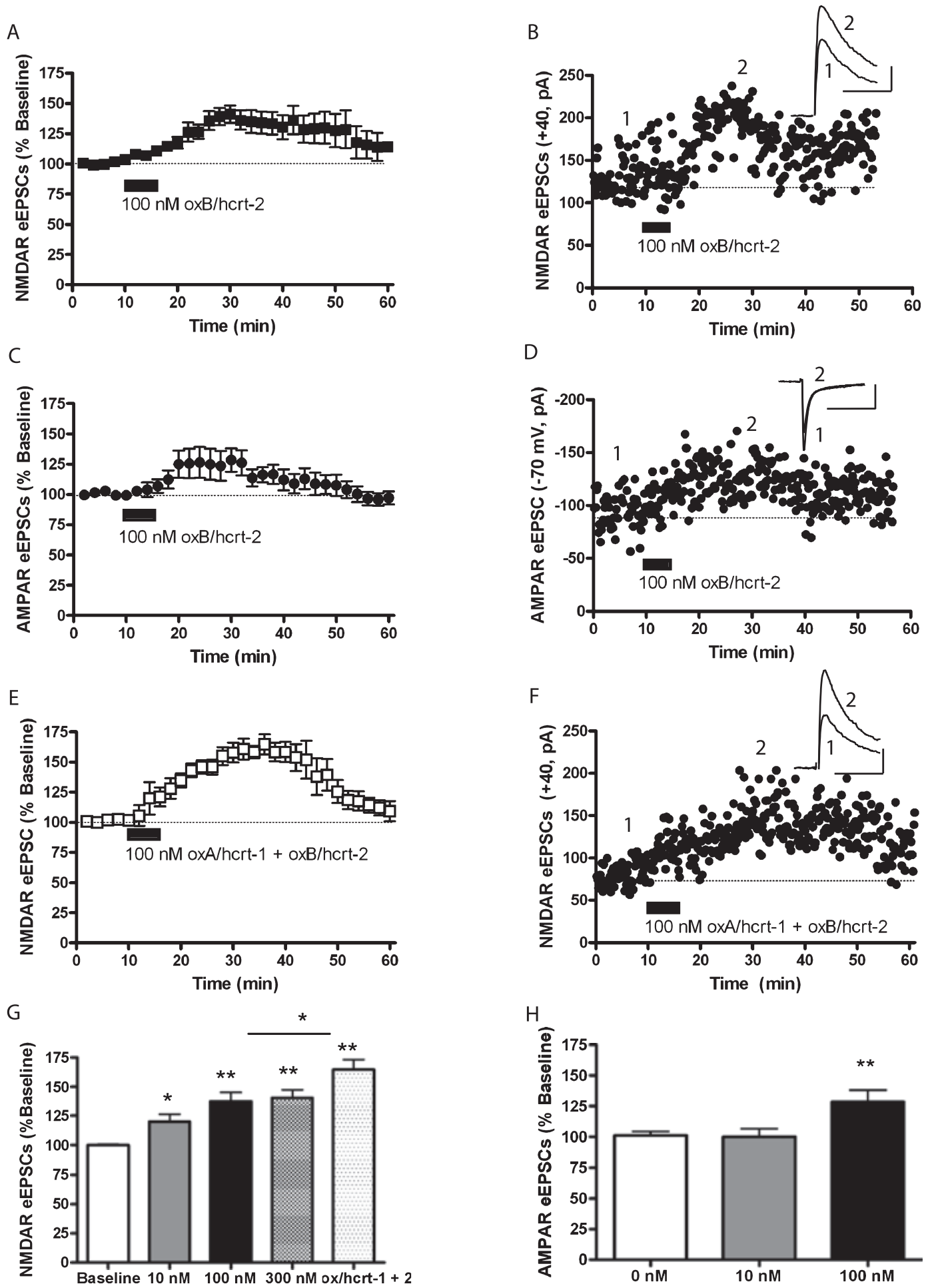

FIG. 1. oxB/hcrt-2 increases excitatory synaptic transmission in VTA neurons. (A) oxB/hcrt-2 (100 nM) increased NMDAR eEPSCs $(n=8)$ evoked at $+40 \mathrm{mV}$. (B) An example recording of NMDAR eEPSCs before, during and after oxB/hcrt-2 application. Inset is an example trace before (1) and after (2) oxB/hcrt-2 application. Scale bars, $50 \mathrm{pA}, 50 \mathrm{~ms}$. (C) oxB/hcrt-2 (100 nM) increases AMPAR eEPSCs evoked at $-70 \mathrm{mV}(n=10)$. (D) An example recording of oxB/hcrt-2 effects on AMPAR-mediated eEPSCs. Inset is an example trace before (1) and after (2) oxB/hcrt-2 application. Scale bars, $50 \mathrm{pA}, 50 \mathrm{~ms}$. (E) oxB/hcrt-2 applied with oxA/hert-1 (100 nM, $5 \mathrm{~min})$ has additive effects on NMDAR currents $(n=8)$. (F) An example recording of NMDAR eEPSCs before, during and after a 5-min application of oxB/hert-2 and oxA/hcrt-1 application. Inset is an example trace before (1) and after (2) oxB/hcrt-2 application. Scale bars, $50 \mathrm{pA}, 50 \mathrm{~ms}$. (G) Maximal potentiation of NMDAR eEPSCs by $10 \mathrm{nM}$ oxB/hcrt-2, $100 \mathrm{nM}$ oxB/hert-2, $300 \mathrm{nM}$ oxB/hcrt-2 or $100 \mathrm{nM}$ oxB/hcrt-2 with oxA/hcrt-1. All treatments were significantly greater than the baseline $(P<0.05$; one-way ANOVA) and both peptides caused significantly greater potentiation of NMDAR eEPSCs than oxB/hcrt-2 alone $(P<0.05$, one-way ANOva, Bonferonni post-test). $(\mathrm{H})$ oxB/hcrt-2 at $100 \mathrm{~nm}$ significantly potentiated AMPAR eEPSCs $(P<0.05)$, but not at $10 \mathrm{~nm}(P<0.05$; one-way ANOVA). Bars represent mean and SEM. $* P<0.05, * * P<0.01$. Stimulus artifact on example traces has been removed for clarity. 
artifact, a time point at which the glutamatergic eEPSC is mediated purely by NMDARs. Bath application of oxB/hcrt-2 (5 min) resulted in a concentration-dependent potentiation of NMDAR-mediated eEPSCs (10 nM: $120 \pm 6 \%, n=7 ; 100 \mathrm{nM:} 138 \pm 8 \%, n=8$; $300 \mathrm{~nm} 140 \pm 7 \%, n=7 ; 19 / 22$ neurons responded to oxB/hcrt-2; Fig. 1A, B and G). To measure AMPAR eEPSCs, VTA neurons were voltage-clamped at $-70 \mathrm{mV}$. oxB/hcrt-2 (100 nM for $5 \mathrm{~min}$ ) also potentiated AMPAR eEPSCs $128 \pm 9 \%$ (Fig. $1 \mathrm{C}$ and D, $n=10$, $P<0.01,8 / 10$ cells responded to oxB/hrct-2). However, a lower concentration of oxB/hcrt-2 (10 nM for $5 \mathrm{~min})$ did not potentiate AMPAR eEPSCs $(n=7, P>0.05$; Fig. $1 \mathrm{H})$. Taken together, these results demonstrate that $\mathrm{oxB} / \mathrm{hcrt}-2$ potentiated AMPAR and NMDAR currents in VTA neurons.

In contrast to oxB/hrct-2, oxA/hcrt-1 potentiated only NMDAR currents in VTA neurons (Borgland et al., 2006). Therefore, we tested whether oxA/hcrt-1 and oxB/hcrt-2, when bath applied together, had additive effects on NMDAR eEPSCs. We found that when a saturating concentration of oxA/hcrt-1 (Uramura et al., 2001; Narita et al., 2007; Fig. 1G) was added with oxB/hcrt-2, the effects were significantly greater than when oxB/hcrt-2 (100 nM) was applied alone $(165 \pm 9 \%$, $n=8, P<0.05$; Fig. $1 \mathrm{E}$ and $\mathrm{F})$, suggesting that oxA/hcrt- 1 and oxB/hcrt-2 exert their actions through distinct receptors, signal transduction pathways and/or cellular targets.

We previously found that oxA/hcrt-1 potentiated NMDAR currents through activation of phospholipase $\mathrm{C}$ (PLC) and PKC (Borgland et al., 2006). Therefore, we next tested if oxB/hcrt-2 potentiated NMDAR eEPSCs through a similar signal transduction mechanism. Inhibitors of PKC were applied intracellularly through the patch pipette. Bisindolylmaleimide (bis; $1 \mu \mathrm{M}$ ) or chelerythrin $(1 \mu \mathrm{M})$ significantly reduced but did not completely block oxB/hcrt-2mediated potentiation of NMDAR eEPSCs compared with untreated neurons (Fig. $2 \mathrm{~A}$ and $\mathrm{B}, P<0.05$ ). oxB/hert- 2 significantly potentiated NMDAR currents $120 \pm 9$ and $120 \pm 13 \%$ in the presence of bis $(1 \mu \mathrm{M} ; n=7, P<0.05)$ or chelerythrin $(1 \mu \mathrm{M} ; n=7, P<0.05)$, respectively. To test if oxB/hcrt-2-mediated potentiation of NMDARs was mediated by protein kinase A (PKA), we applied PKI $(20 \mu \mathrm{M})$ through the patch pipette. In the presence of PKI, oxB/hcrt-2 potentiation of NMDARs was not significantly different from that of untreated VTA neurons $(138 \pm 14 \%, n=6, P>0.05$, Fig. $2 \mathrm{C}$ and D). To determine whether potentiation of AMPAR eEPSCs by oxB/hort- 2 occurred through activation of PKC, we recorded AMPAR eEPSCs with bis $(1 \mu \mathrm{M})$ or chelerythrin $(1 \mu \mathrm{M})$ in the patch pipette. Neither bis nor chelerythrin had effects on $\mathrm{oxB} /$ hcrt-2-mediated potentiation of AMPAR eEPSCs when applied intracellularly directly to the postsynaptic neuron (bis: $128 \pm 9, n=5$; chelerythrin: $128 \pm 11 \%, n=8$; Fig. 2E and F). Because oxB/hcrt-2 potentiation of NMDAR currents was partially mediated by $\mathrm{PKC}$ signaling and $\mathrm{oxB} / \mathrm{hcrt}-2$ potentiation of AMPAR currents was not, we hypothesized that oxB/hcrt-2 may be exerting both a presynaptic increase of glutamate release and a postsynaptic PKC-dependent potentiation of NMDARs. To determine if oxB/hcrt-2 was signaling via PKC presynaptically to increase glutamate release, we soaked slices in the $\mathrm{PKC}$ inhibitor bis $(10 \mu \mathrm{M})$ for $45 \mathrm{~min}$ prior to the application of oxB/hcrt-2 (100 nM). oxB/hcrt- 2 potentiation of NMDARs $(n=7$; Fig. $2 \mathrm{G})$ or AMPARs was abolished in the presence of externally applied bis $(n=7$; Fig. $2 \mathrm{H}$ ). These results suggest that $\mathrm{oxB} / \mathrm{hcrt}-2$ signals presynaptically through a PKC-dependent mechanism.

To verify further if oxB/hcrt-2-mediated potentiation of AMPARs was mediated pre- or post-synaptically, we examined the effects of $\mathrm{oxB} /$ hcrt- 2 on the probability of transmitter release, comparing the response to paired pulses, a measure that changes in a highly predictable fashion with release probability (Zucker \& Regehr, 2002).
We recorded the effect of oxB/hcrt-2 (100 nM, $5 \mathrm{~min})$ on AMPAR eEPSCs at $-70 \mathrm{mV}$ using a paired-pulse stimulation protocol with a 50 -ms interval. Fifteen minutes after bath application, oxB/hcrt-2 increased the amplitude of the first eEPSC in the paired-pulse protocol (126 $\pm 12 \%$ of basal, $P<0.05$, data not shown), but did not significantly alter the second eEPSC. Application of oxB/hcrt-2 significantly decreased the ratio between the two pulses (Fig. 3A and B; $n=8, P<0.01$ ), suggesting that $\mathrm{oxB} / \mathrm{hcrt}-2$ increases the probability of transmitter release.

Next, we further examined the locus of synaptic change by measuring the frequency and amplitude of AMPAR mEPSCs recorded when neurons were voltage clamped at $-70 \mathrm{mV}$. Although oxB/hcrt- 2 had no effect on the amplitude of mEPSCs (control: $13.0 \pm 0.8, n=6$; oxB/hcrt-2: $12.6 \pm 0.6, n=6, P>0.05$; Fig $3 \mathrm{D}, \mathrm{E}$ and $\mathrm{G})$, the frequency of $\mathrm{mEPSCs}$ was significantly greater than in controls (control: $1.0 \pm 0.2 \mathrm{~Hz}, n=6$, oxB/hcrt-2: $1.4 \pm 0.2, n=6, P<0.05$; Fig $3 \mathrm{C}, \mathrm{E}$ and F). Taken together, these results indicate that oxB/hcrt- 2 can increase the probability of glutamate release at VTA synapses.

\section{Activation of OX2/hcrt-2 receptors potentiates NMDAR but not AMPAR currents}

oxB/hcrt-2 interacts mainly with $\mathrm{OX} 2 / \mathrm{Hcrt}-2$ receptors, although it also has low affinity for OX1/Hcrt-1 receptors (Sakurai et al., 1998). In the next series of experiments, we attempted to determine through which receptor $\mathrm{oxB} / \mathrm{hcrt}-2$ acted to increase glutamatergic synaptic transmission. To determine if oxB/hcrt-2 potentiated NMDAR EPSCs via activation of $\mathrm{OX} 1 / \mathrm{Hcrt}-1$ receptors, we bath applied oxB/hcrt-2 $(100 \mathrm{nM})$ in the presence of the OX1/Hcrt-1 receptor antagonist SB 334867 ( $1 \mu \mathrm{M}$; Fig. 4A). oxB/hcrt-2 potentiated NMDAR eEPSCs by $128 \pm 7 \%(n=7$, Fig. 4A). This effect was not significantly different from NMDAR potentiation by oxB/hert-2 alone $(P>0.05$, Fig. $1 \mathrm{G})$. We tested if oxB/hcrt-2 could potentiate NMDAR eEPSCs in the presence of a putative OX2/hcrt-2 receptor antagonist, JNJ-10397049, which has 600 -fold selectivity for OX2/Hcrt-2 over OX1/Hcrt-1 (McAtee et al., 2004). The putative OX2/Hcrt-2 receptor antagonist significantly inhibited $\mathrm{oxB} /$ hcrt-2-mediated potentiation of NMDAR eEPSCs $(117 \pm 5, n=7, P<0.05$; Fig. 4B). Additionally, we found that NMDAR-mediated potentiation by $10 \mathrm{nM}$ oxB/hcrt-2, a concentration that does not potentiate AMPARs, could be completely blocked by JNJ-10397049 ( $1 \mu \mathrm{M} ; n=6$; Fig. 4C), suggesting that the postsynaptic effects of oxB/hcrt- 2 on NMDAR currents occur through activation of OX $2 / \mathrm{Hcrt}-2$ receptors. To confirm that NMDAR eEPSCs potentiated via activation of $\mathrm{OX} 2 / \mathrm{Hcrt}-2$ receptors, we applied the highly selective OX2/Hcrt-2 agonist Ala ${ }^{11}$-D-Leu ${ }^{15}$-orexin $B$ to VTA slices. Bath-applied $\mathrm{Ala}^{11}$-D-Leu ${ }^{15}$-orexin B (10 nM) significantly increased NMDAR eEPSCs in VTA neurons (Fig. 4D, $166 \pm 20 \%, n=7, P<0.01,6 / 7$ neurons responded). This effect was completely blocked by the putative OX2/Hcrt-2 receptor antagonist JNJ-10397049 (Fig. 4E; $n=5$ ). To determine if a purely post-synaptic effect of oxB/hcrt-2 could be blocked by the OXR2 antagonist, we measured the response of post-synaptic NMDARs to a submaximal concentration of NMDA $(20 \mu \mathrm{M}, 30 \mathrm{~s})$ before and $10 \mathrm{~min}$ after a 5 -min bath application of oxB/hcrt-2 in the presence or absence of JNJ-10397049 $(1 \mu \mathrm{M})$. oxB/hcrt-2 significantly potentiated the response to NMDA (before: $88 \pm 10 \mathrm{pA}$, after: $139 \pm 18 \mathrm{pA}$, Fig. $4 \mathrm{~F}, n=7, P<0.05)$. In the presence of JNJ-10397049, the NMDA-mediated change in holding current was not significantly different after oxB/hcrt-2 application (before: $90 \pm 1 \mathrm{pA}$, after: $91 \pm 12$ pA, Fig. $4 \mathrm{H}, n=7, P>0.05)$. To determine which receptor was responsible for the presynaptic effects of oxB/hcrt-2 on AMPAR 
A

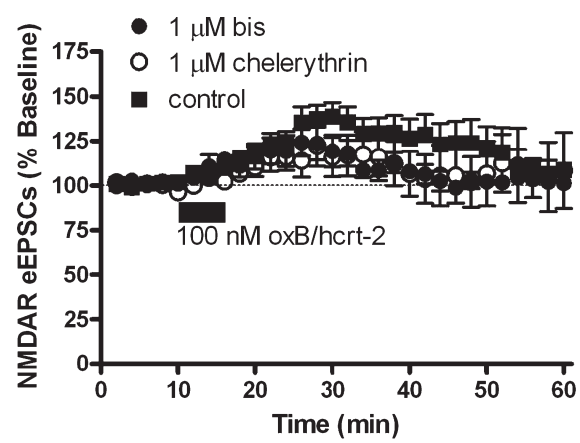

C

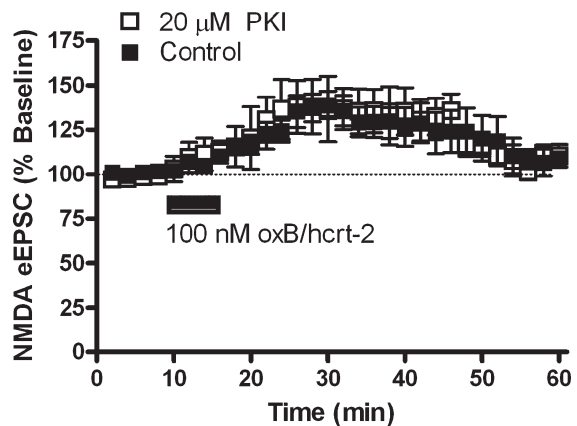

$\mathrm{E}$

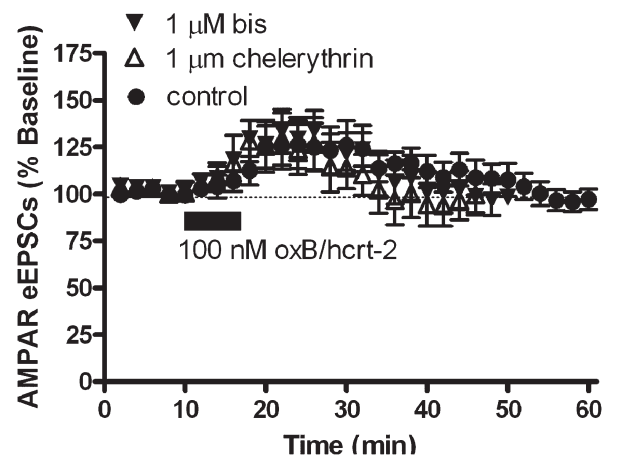

G

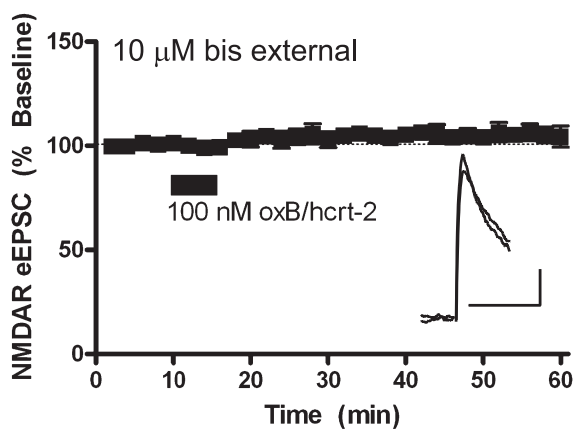

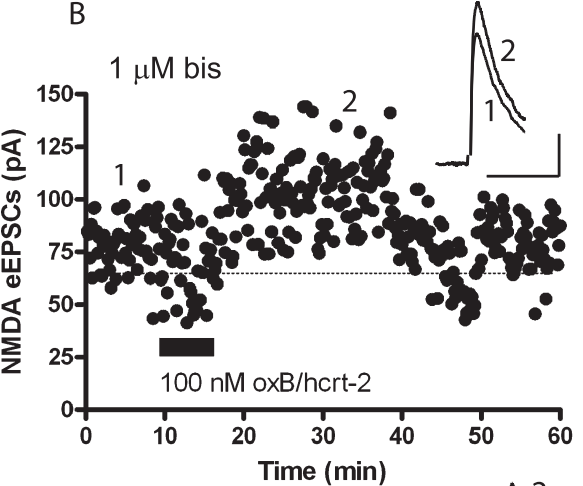
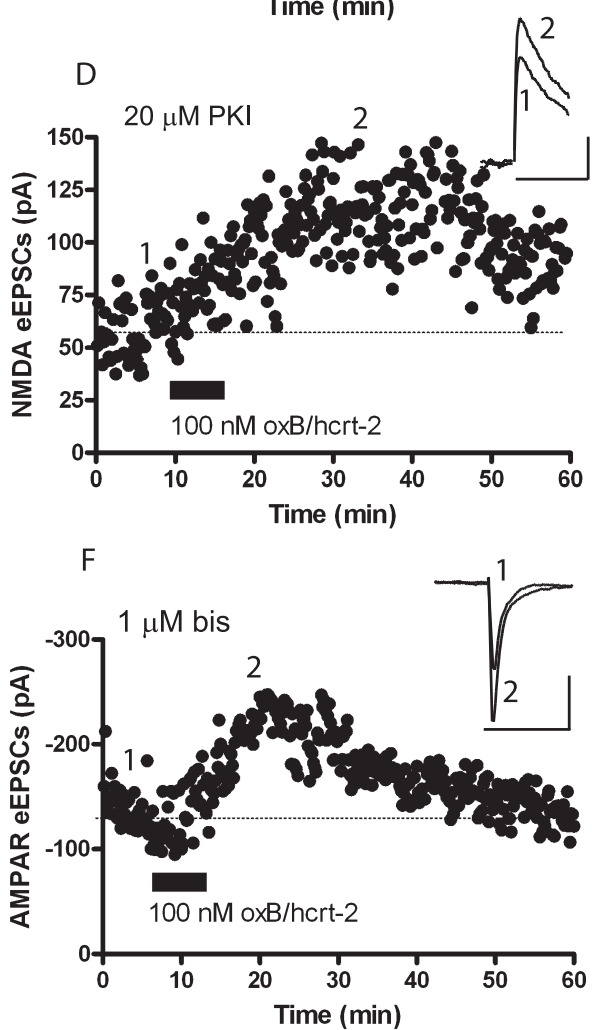

$\mathrm{H}$

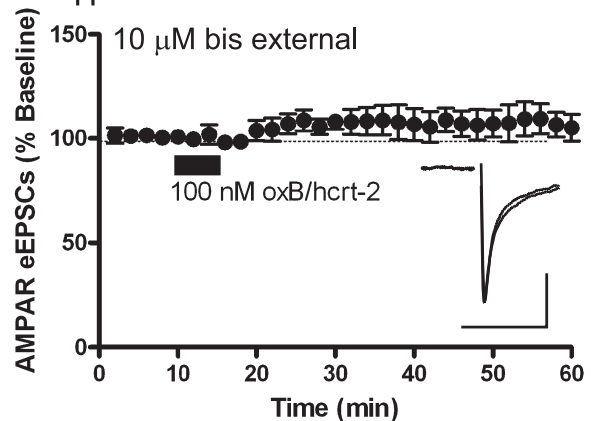

FIG. 2. oxB/hcrt-2-mediated potentiation of glutamatergic synaptic transmission is dependent on PKC signaling. (A) PKC inhibitors, either bisindolylmaleimide (bis, $1 \mu \mathrm{M}, n=7)$ or chelerythrin $(1 \mu \mathrm{M}, n=7)$ reduced oxB/hcrt-2- mediated potentiation of NMDAR eEPSCs in VTA neurons. (B) An example recording of NMDAR eEPSCs before, during and after a 5-min application of oxB/hcrt-2 in the presence of intracellularly administered bis $(1 \mu \mathrm{M})$. Inset is an example trace before (1) and after (2) oxB/hert-2 application. Scale bars, $50 \mathrm{pA}, 50 \mathrm{~ms}$. (C) oxB/hcrt-2-mediated potentiation of NMDARs is not altered by intracellularly applied PKA inhibitor, PKI $(20 \mu \mathrm{M} ; n=6)$. (D) An example recording of oxB/hert-2 potentiation of NMDAR eEPSCs in the presence of PKI. Inset is an example trace before (1) and after (2) oxB/hcrt-2 application. Scale bars, $50 \mathrm{pA}, 50 \mathrm{~ms}$. (E) AMPAR eEPSCs were potentiated by oxB/hcrt-2 in the presence of intracellularly applied bis $(1 \mu \mathrm{M}, n=5)$ or chelerythrin $(1 \mu \mathrm{M}, n=8)$. (F) An example recording of AMPAR currents evoked at $-70 \mathrm{mV}$ in the presence of bis $(1 \mu \mathrm{M})$ before, during and after oxB/hcrt-2 (100 nM, $5 \mathrm{~min})$ application. Inset is an example trace before (1) and after (2) oxB/hcrt-2 application. Scale bars, $50 \mathrm{pA}, 20 \mathrm{~ms}$. (G) $\mathrm{oxB} /$ hcrt-2 $(100 \mathrm{nM})$ did not significantly potentiate NMDARs in the presence of externally applied bis $(10 \mu \mathrm{M}, n=7)$. Inset is an example trace before and after oxB/hcrt-2 application. Scale bars, $50 \mathrm{pA}, 20 \mathrm{~ms}$. (H) oxB/hcrt-2 $(100 \mathrm{nM})$ did not significantly potentiate AMPARs in the presence of externally applied bis $(10 \mu \mathrm{M}, n=7)$. Inset is an example trace before and after oxB/hcrt-2 application. Scale bars, $50 \mathrm{pA}, 20 \mathrm{~ms}$. Stimulus artifact on example traces has been removed for clarity. 
A

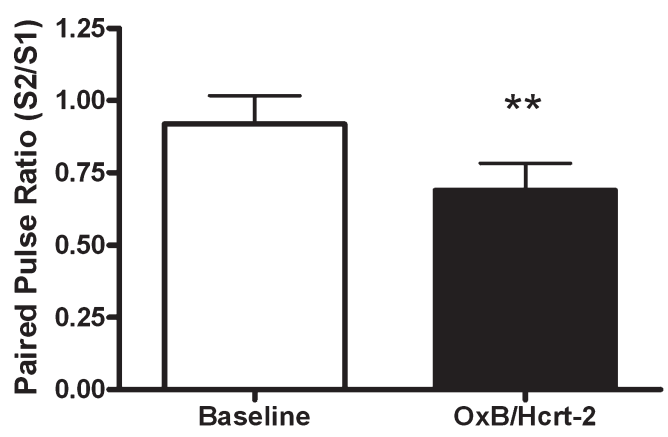

C

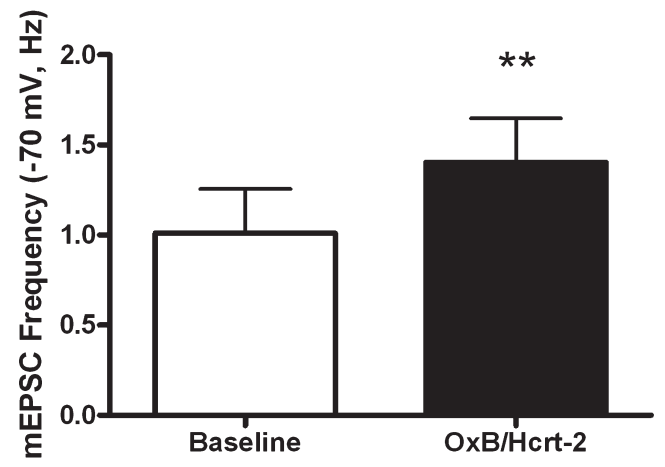

$\mathrm{E}$ Baseline

F

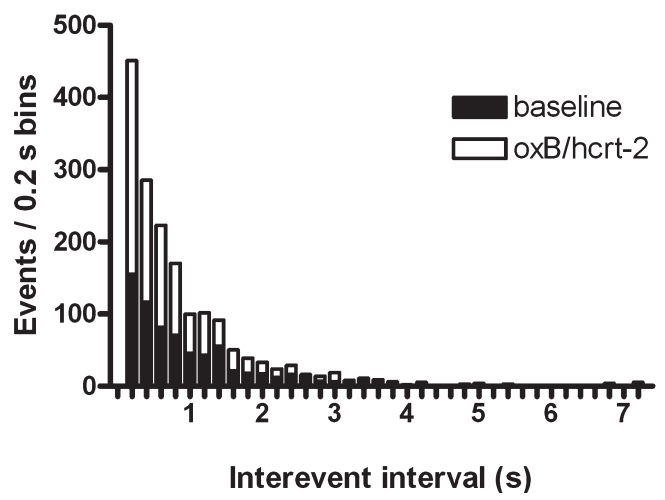

B

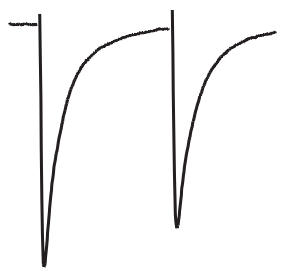

Baseline

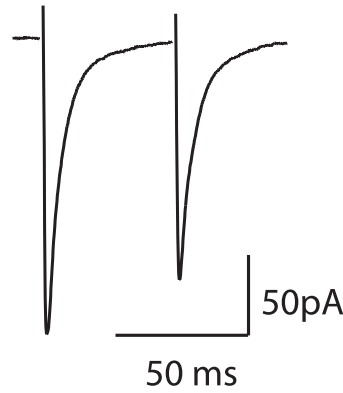

OxB/Hcrt-2

$100 \mathrm{nM}$

D $\quad 50 \mathrm{~ms}$

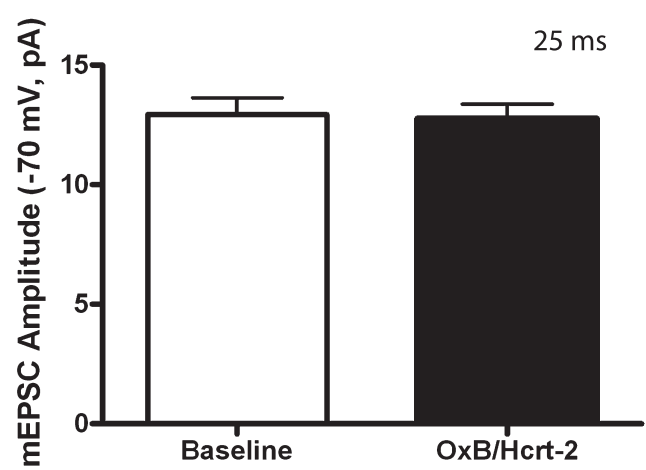

OxB/Hcrt-2

$100 \mathrm{nM}$

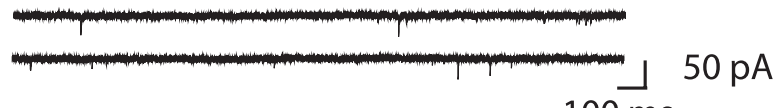

G

$100 \mathrm{~ms}$

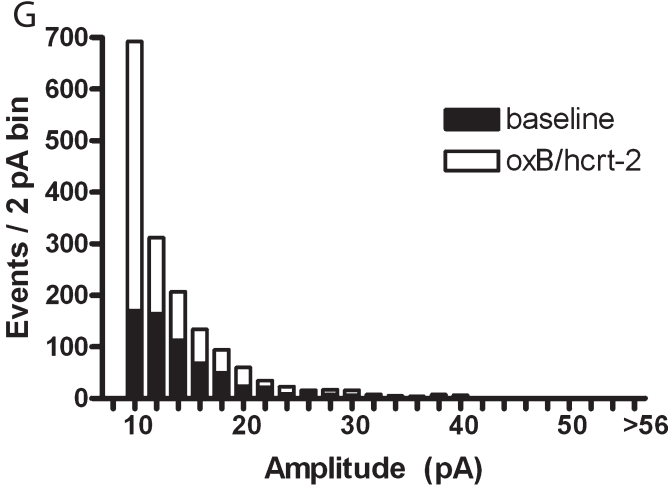

FIG. 3. oxB/hcrt-2 causes presynaptic glutamate release. (A) A paired-pulse protocol using a 50 -ms inter-stimulus interval showed depressed ratio after application of oxB/hcrt-2 (100 nM; $n=9 ; P<0.01)$. (B) An example trace of a neuron recorded before (upper panel) and $10 \mathrm{~min}$ after a 5 -min oxB/hert-2 application (lower panel). Stimulus artifact has been removed for clarity. (C) oxB/hort-2 $(100 \mathrm{nM} ; n=7)$ induced a significant increase in $\mathrm{mEPSC}$ frequency $(n=7 ; P<0.05$, paired $t$-test). (D) mEPSC amplitude was unchanged after oxB/hcrt-2 application (100 nM; $n=7 ; P>0.05$, paired $t$-test). Bars represent mean and SEM (** $P<0.01)$. (E) Example of mEPSCs before (left) and $10 \mathrm{~min}$ after (right) oxB/hcrt-2 (100 nM, $5 \mathrm{~min}$ ) treatment. (F) Frequency histogram of amplitudes from all mEPSCs from before $(n=7)$ or $10 \mathrm{~min}$ after oxB/hert-2 treatment $(n=7)$. (G) Frequency histogram of inter-event intervals of all $\mathrm{mEPSCs}$ from before $(n=7)$ or $10 \mathrm{~min}$ after oxB/hort-2 treatment $(n=7)$.

eEPSCs, we tested whether oxB/hcrt-2 potentiation of AMPARs could be blocked by $\mathrm{OX} 1 / \mathrm{Hcrt}-1$ or $\mathrm{OX} 2 / \mathrm{Hcrt}-2$ receptor antagonists. Surprisingly, neither SB $334867(1 \mu \mathrm{M}, 123 \pm 7 \%, n=6$;
Fig. 5A) nor JNJ-10397049 (1 $\mu \mathrm{M}, 125 \pm 7 \%, n=7$; Fig. 5B) significantly blocked $\mathrm{oxB} / \mathrm{hcrt}-2$-mediated potentiation of AMPAR currents. Furthermore, bath application of the selective OX2/Hcrt-2 

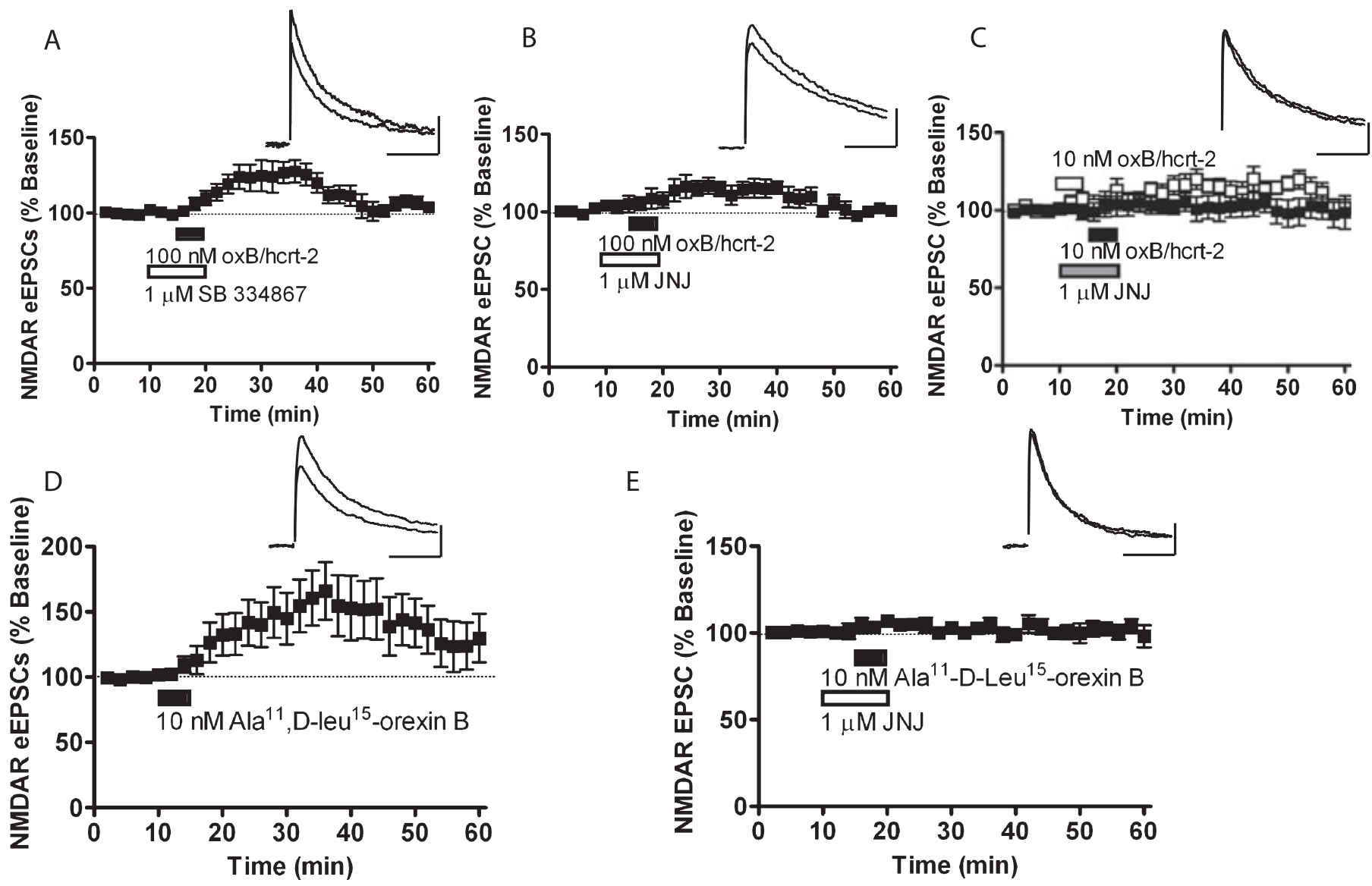

$\mathrm{E}$

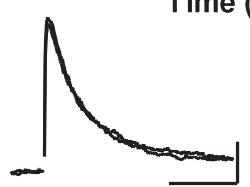

$\mathrm{F}$

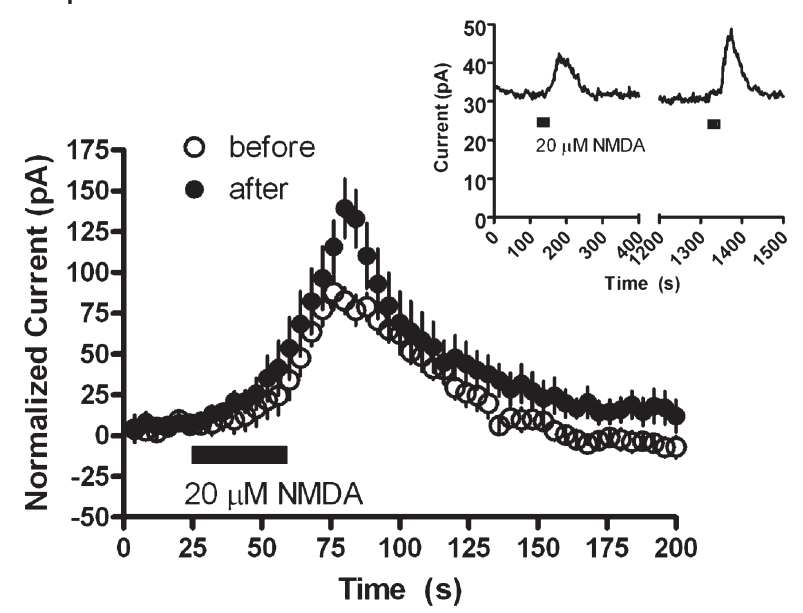

G

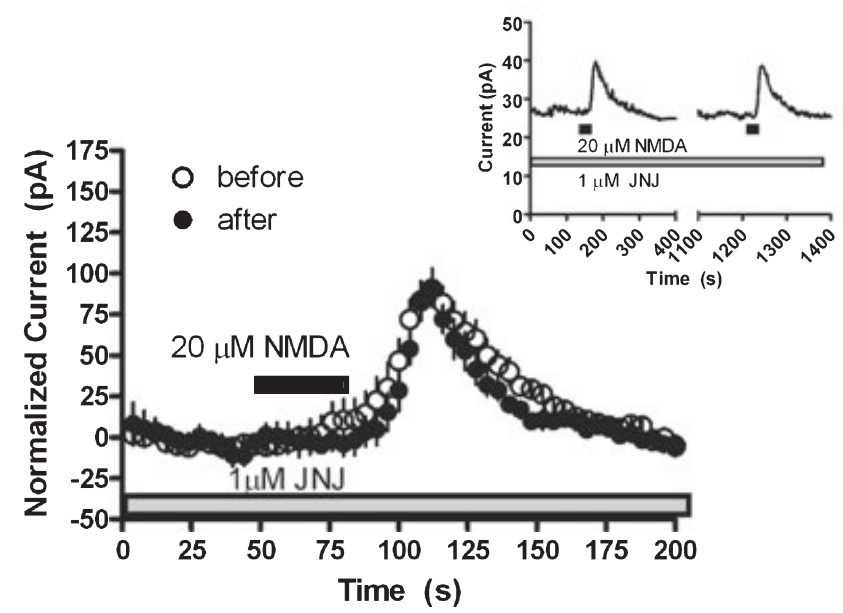

FIG. 4. Potentiation of post-synaptic NMDAR-mediated synaptic transmission by OX2/Hcrt-2 receptors. (A) oxB/hcrt-2 (100 nM, 5 min) potentiated NMDAR eEPSCs in the presence of the selective OX1/Hcrt-1 receptor antagonist SB $334867(1 \mu \mathrm{M}, 10 \mathrm{~min}, n=7)$. Inset shows an example trace before and after oxB/hcrt2 application. Scale bars, $50 \mathrm{pA}, 50 \mathrm{~ms}$. (B) The OX2/Hcrt-2 receptor antagonist JNJ-10397049 (1 $\mu \mathrm{M})$ partially blocked NMDAR-mediated potentiation by $100 \mathrm{nM} \mathrm{oxB} / \mathrm{hcrt}-2(n=7)$. Inset shows an example trace before and after oxB/hcrt-2 application. Scale bars, $50 \mathrm{pA}, 50 \mathrm{~ms}$. (C) oxB/hcrt-2 at $10 \mathrm{~nm}$ did not potentiate NMDAR eEPSCs in the presence of JNJ-10397049 $(1 \mu \mathrm{M})$. Open squares reflect NMDAR potentiation by $10 \mathrm{nM}$ oxB/hcrt-2 $(n=7)$. Filled squares represent oxB/hcrt-2 application in the presence of $1 \mu \mathrm{M} \mathrm{JNJ}-1039749(n=6)$. Inset shows an example trace before and after oxB/hcrt-2 application. Scale bars, $50 \mathrm{pA}, 50 \mathrm{~ms}$. (D) The selective OX2/Hcrt-2 receptor agonist Ala ${ }^{11}$-D-Leu ${ }^{15}$-orexin B $(10 \mathrm{nM})$ increased NMDAR eEPSCs recorded at $+40 \mathrm{mV}(n=7,6 / 7 \mathrm{cells}$ responded). Inset shows an example trace before and after oxB/hcrt-2 application. Scale bars, $50 \mathrm{pA}, 50 \mathrm{~ms}$. (E) JNJ-10397049 (1 $\mu \mathrm{M})$ blocked Ala ${ }^{11}$-D-Leu ${ }^{15}$ orexin B- $(10 \mathrm{nM})$ mediated potentiation of NMDARs $(n=5)$. Inset shows an example trace before and after oxB/hcrt-2 application. Scale bars, $50 \mathrm{pA}, 50 \mathrm{~ms}$. (F)

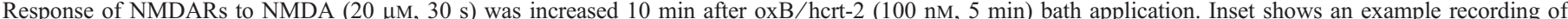
NMDA-mediated change in current before and after oxB/hcrt-2 (100 nM). (G) In the presence of JNJ-10397049 (1 $\mu$ M), the response of NMDARs to NMDA $(20 \mu \mathrm{M}, 30 \mathrm{~s})$ was not potentiated $10 \mathrm{~min}$ after oxB/hcrt-2 (100 nM, $5 \mathrm{~min})$ application. Inset shows an example recording of NMDA-mediated change in current before and after oxB/hcrt-2 (100 nM) in the presence of JNJ-10397049 $(1 \mu \mathrm{M})$. 

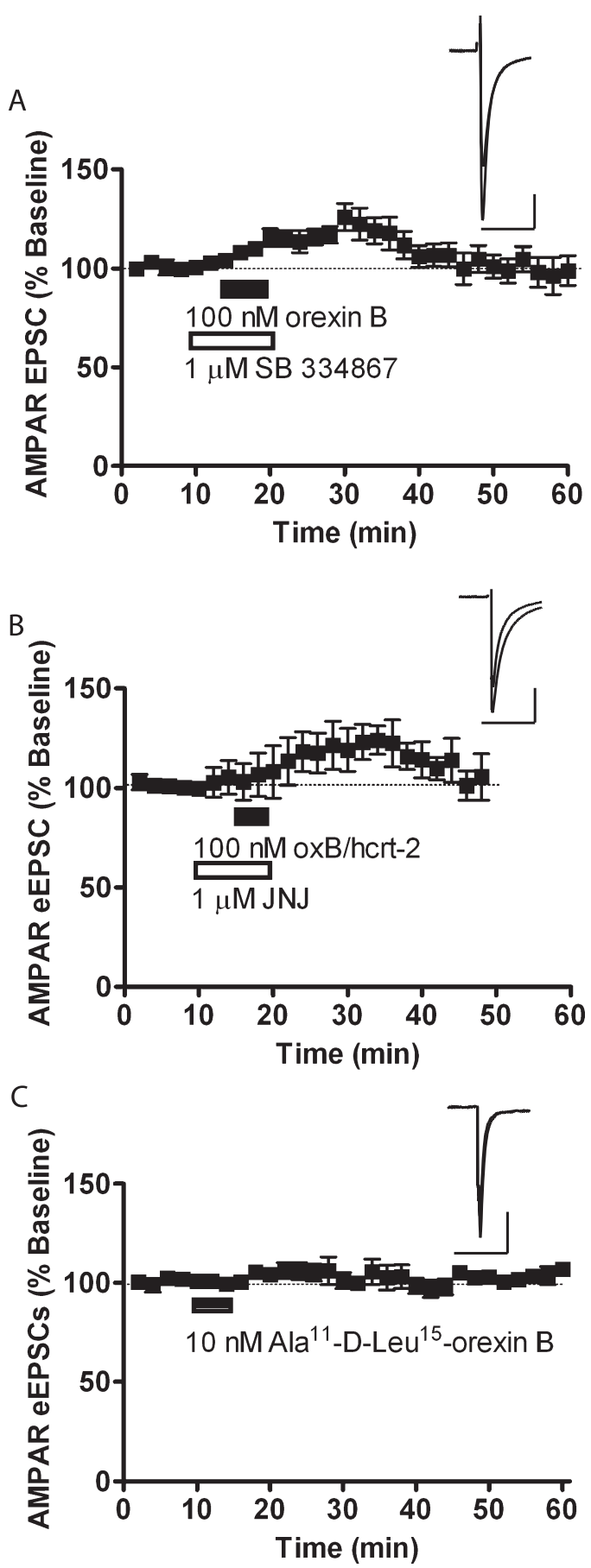

FIG. 5. oxB/hcrt-2 potentiation of AMPARs is not blocked by OXR1/Hert-1 or OXR2/Hcrt-2 antagonists. (A) oxB/hcrt-2 (100 nM) potentiated AMPAR eEPSCs in the presence of the OX1/Hcrt-1 receptor antagonist SB 334867 $(1 \mu \mathrm{M}, n=6)$. Inset shows an example trace before and after oxB $/$ hcrt-2 application. Scale bars, $50 \mathrm{pA}, 20 \mathrm{~ms}$. (B) In the presence of the OX2/Hcrt-2 receptor antagonist JNJ-10397049 (1 $\mu \mathrm{M}, n=7)$, oxB/hcrt-2 (100 nM) potentiated AMPAR eEPSCs. Inset shows an example trace before and after oxB/hcrt-2 application. Scale bars, 50 pA, $20 \mathrm{~ms}$. (C) $\mathrm{Ala}^{11}$-D-Leu ${ }^{15}$-orexin B $(10 \mathrm{nM})$ did not increase AMPAR eEPSCs $(n=7)$. Inset shows an example trace before and after oxB/hcrt-2 application. Scale bars, 50 pA, $20 \mathrm{~ms}$.

receptor agonist $\mathrm{Ala}^{11}$-D-Leu ${ }^{15}$-orexin $\mathrm{B}(10 \mathrm{nM})$ did not potentiate AMPAR currents (Fig. 5C, $n=7$ ). Taken together, these results suggest that $\mathrm{xB} /$ hcrt-2 can potentiate NMDAR, but not AMPAR currents via activation of $\mathrm{OX} 2 / \mathrm{Hcrt}-2$ receptors, and the receptor responsible for the $\mathrm{oxB} / \mathrm{hcrt}-2$-mediated presynaptic effect remains unknown.

\section{oxB/hcrt-2 potentiation of NMDARs inhibits LTP}

We have demonstrated that oxB/hcrt-2 can increase transmitter release and cause a postsynaptic potentiation of NMDARs. LTP in the VTA is dependent on activation of NMDARs (Bonci \& Malenka, 1999; Overton et al., 1999). Therefore, we wanted to determine if oxB/hcrt-2 activation of NMDARs or oxB/hcrt-2-mediated increases in intracellular $\mathrm{Ca}^{2+}$ release in the VTA (Uramura et al., 2001) could alter LTP in VTA slices. To induce LTP, we used a spike-timing protocol consisting of bursts of EPSP-spike pairs, where the onset of EPSPs precedes the postsynaptic spike by $5 \mathrm{~ms}$ (Liu et al., 2005). This pairing protocol reliably induces an NMDAR-dependent LTP in VTA dopamine neurons (Liu et al., 2005). EPSP-spike pairing induced robust LTP in untreated rat VTA neurons reaching a maximum of $141 \pm 7 \%(n=11$; Fig. $6 \mathrm{~A}$ and D). Pre-treatment of oxB $/$ hert-2 $(100 \mathrm{nM}$ for $5 \mathrm{~min})$ induced a potentiation of AMPAR EPSPs (Fig. 6B). Interestingly, only a small, non-significant potentiation could be induced with the pairing protocol after oxB/hcrt- 2 potentiated AMPARs $(120 \pm 7 \%, n=11$; Fig. $6 \mathrm{~B}$ and $\mathrm{D})$. We tested if $\mathrm{Ala}^{11}$-D-Leu ${ }^{15}$-orexin $\mathrm{B}$, which only potentiates NMDARs but not AMPARs, could occlude LTP. We bath applied Ala ${ }^{11}$-D-Leu ${ }^{15}$-orexin $\mathrm{B}(10 \mathrm{nM})$ for $5 \mathrm{~min}$ and then induced LTP with the pairing protocol 10 min after washout. EPSP-spike pairing-induced LTP was significantly less than in untreated VTA neurons $(117 \pm 4, n=7, P<0.05$; Fig. $6 \mathrm{C}$ and $\mathrm{D})$. Taken together, these results indicate that significantly less LTP was induced after oxB/hcrt-2 activation of NMDAR currents in VTA neurons.

To verify if oxB/hcrt-2 inhibition of LTP occurred in dopaminergic neurons of the VTA, we filled neurons with biocytin while recording EPSPs and subsequently processed slices for TH immunocytochemistry, a marker for dopamine neurons. Of 11 neurons recorded, nine were stained for TH (Fig. 6E).

\section{Discussion}

\section{oxB/hcrt-2 increases presynaptic glutamate release in the VTA}

Our findings demonstrate that oxB/hort-2 enhances glutamatergic synaptic transmission in the VTA through action at both pre- and postsynaptic sites. Two peptide products are released from the cleavage of pre-pro-orexin/hypocretin: oxA/hcrt-1 and oxB/hcrt-2. oxA/hcrt-1 is more stable in blood and cerebrospinal fluid (Kastin \& Akerstrom, 1999). However, central nervous system oxB/hcrt-2 levels are consistently 2-5 times higher than oxA/hcrt-2 levels (Mondal et al., 1999a,b; Date et al., 2000a,b). Both peptides have action in the VTA in that both increase firing in dopaminergic neurons (Korotkova et al., 2003) and, when micro-injected in the VTA, increase dopamine release in the nucleus accumbens (Narita et al., 2006, 2007). Additionally, in a previous study, we found that oxA/hcrt-1 potentiated NMDARs but not AMPARs in VTA dopamine neurons via activation of OX1/Hcrt-1 receptors and PKC (Borgland et al., 2006). Here, oxB/hcrt-2 played a distinct role from oxA/hert-1, as oxB/hcrt-1 potentiated both AMPAR and NMDAR currents in VTA slices. oxB/hcrt-2 increased mEPSC frequency, but not amplitude, and caused paired-pulse depression of AMPAR currents, two measures that are highly indicative of presynaptic glutamate release. Furthermore, oxB/hcrt-2-mediated potentiation of AMPAR currents was blocked only by external application of the PKC inhibitor bis, but not intracellularly applied bis. These data suggest that 

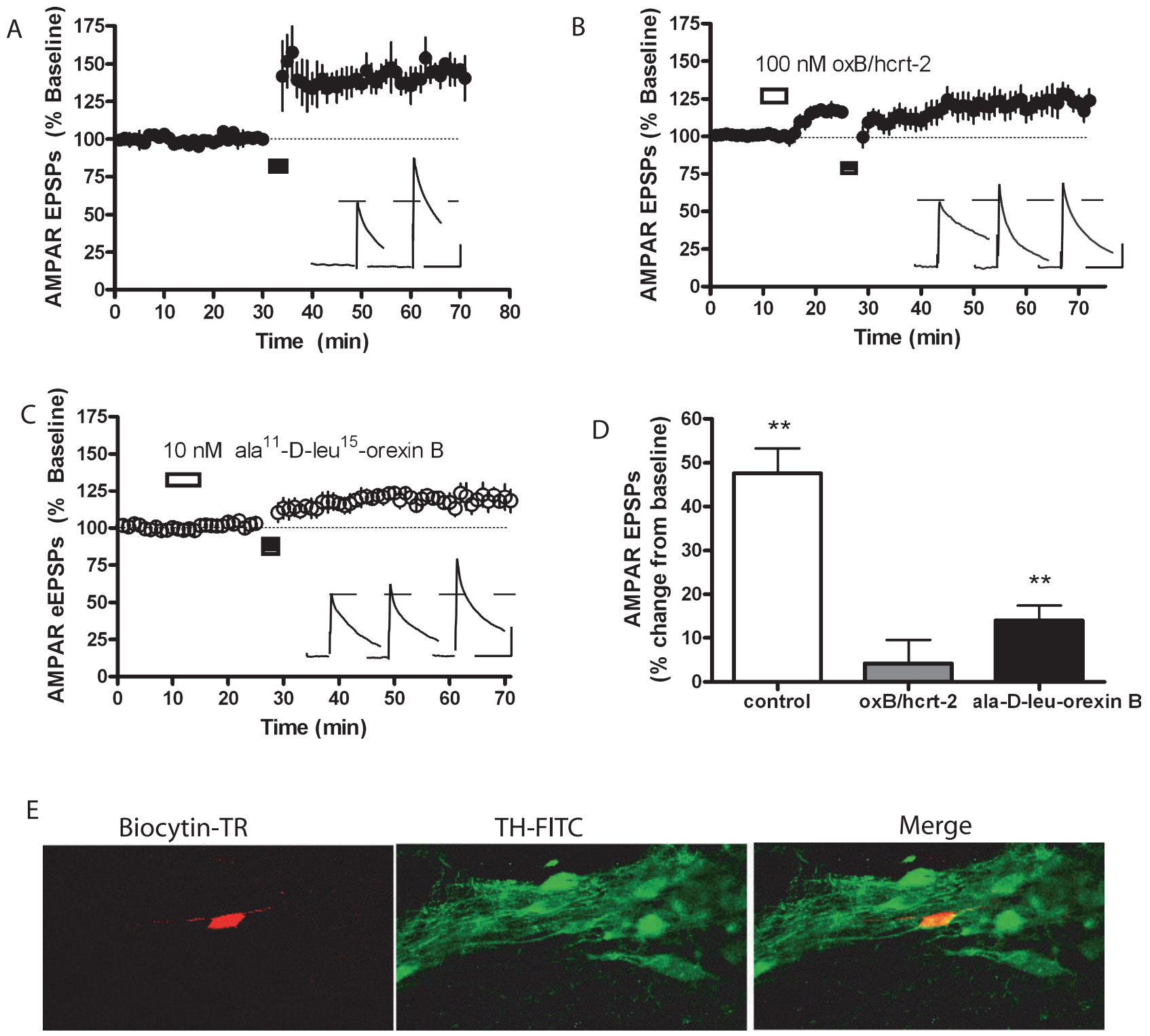

FIG. 6. oxB/hcrt-2 partially occludes LTP in VTA dopamine neurons. The protocol for LTP induction consisted of 20 bursts of EPSP-spike pairs. (A) Paired stimulation induced LTP in VTA neurons $(n=11)$. (B) Pretreatment of oxB/hcrt-2 (100 nM, $5 \mathrm{~min})$ potentiated AMPAR EPSPs. Further potentiation could not be induced after EPSP-spike pairing $(n=11)$. (C) LTP was reduced after application of Ala ${ }^{11}$-D-Leu ${ }^{15}$-orexin B $(10 \mathrm{nM} ; n=7)$. (D) Summary of LTP induction in untreated VTA neurons $(n=11)$, neurons treated with oxB/hcrt-2 $(n=11)$ or Ala ${ }^{11}$-D-Leu ${ }^{15}$-orexin B-treated neurons $(n=7)$. AMPAR EPSPs were significantly elevated after the pairing protocol in naïve and $\mathrm{Ala}^{11}$-D-Leu ${ }^{15}$-orexin B-treated neurons $(P<0.001)$, but not oxB/hcrt-2-treated neurons $(P>0.05)$. Bars represent mean and SEM. One-way ANOVA with a Bonferroni post test $(* * P<0.01)$. (E) Slices from LTP experiments were fixed and processed for the presence or absence of $\mathrm{TH}$ and biocytin. oxB/hcrt-2 partially occluded LTP in TH-positive neurons $(9 / 11$ neurons were TH positive). TH was labeled with anti-TH antibodies and FITC. Biocytin was labeled with streptavidin-conjugated Texas Red.

$\mathrm{oxB} /$ hcrt-2 potentiation of AMPAR currents is due to a PKC-mediated increase in pre-synaptic glutamate release. PKC-mediated increases in presynaptic excitatory synaptic transmission were initially observed in the hippocampus (Malenka et al., 1986; Terrian et al., 1991); however, this is the first observation of PKC-mediated increase in glutamate release in the VTA.

Surprisingly, the oxB/hcrt-2-mediated increase in presynaptic glutamate release was not due to activation of OX1/Hcrt-1 or OX2/Hcrt-2 receptors, as neither antagonists SB334867 nor JNJ10397049 blocked oxB/hcrt-2-mediated increase in AMPA currents. Furthermore, the selective OX2/hcrt- 2 agonist did not potentiate AMPAR currents. Enhanced firing of VTA neurons by oxB/hert-2 (Korotkova et al., 2003) could result in increased somatodendritic release of unknown peptides or neuromodulators that may act presynaptically to increase the probability of transmitter release. However, this is unlikely, as oxB/hcrt- 2 enhanced AMPAR mEPSC frequency in the presence of lidocaine, which blocks action potentials. The enhanced presynaptic effect could be due to oxB/hcrt- 2 or a metabolite acting at an unidentified PKC-coupled receptor. Further experiments with other antagonists or receptor knockout mice are required to determine the exact pharmacology of oxB/hcrt-2-mediated potentiation of glutamate release.

A presynaptic effect of oxB/hcrt-2 has been observed in other brain regions. In mouse laterodorsal tegmental neurons, oxB/hcrt-2 elevated mEPSC frequency (Burlet et al., 2002). Furthermore, in rat embryonic hypothalamic neurons in culture, oxB/hcrt-2 increased inhibitory post-synaptic current and EPSC frequency in a tetrodotoxininsensitive manner (Van den pol et al., 1998). 


\section{oxB/hcrt-2 potentiates NMDARs in VTA neurons}

In addition to the oxB/hcrt-2-mediated increase in transmitter release probability, oxB/hcrt-2 also potentiated postsynaptic NMDAR currents. This effect was independent of the presynaptic action of $\mathrm{oxB} / \mathrm{hcrt}-2$, as evidenced by the reduction of NMDAR potentiation with intracellular application of PKC inhibitors, whereas the oxB/hcrt-2-mediated increase in AMPAR synaptic transmission was unaffected. OX2/Hcrt-2 receptors expressed on the VTA dopamine neurons can couple to $\mathrm{G} \alpha \mathrm{q}$ or $\mathrm{G} \alpha \mathrm{i}$ proteins and enhance intracellular calcium via $\mathrm{G} \alpha \mathrm{q}$ or $\mathrm{G} \beta \gamma$ activation of phospholipase $\mathrm{C}$ and $\mathrm{PKC}$ (Uramura et al., 2001; Narita et al., 2007). Furthermore, the postsynaptic current induced by NMDA application was increased after oxB/hcrt-2 application, albeit that the increase induced by oxB/hcrt-2 was smaller than that observed with evoked NMDAR EPSCs. This difference may be attributed to the fact that the evoked recordings examine the effects of both oxB/hcrt-2-induced presynaptic glutamate release on NMDARs in addition to oxB/hcrt-2-mediated postsynaptic receptor potentiation, whereas the bath application of NMDA experiment measures only the latter. Moreover, post-synaptic effects of oxB/hcrt-2 may result in potentiation of selective subtypes of postsynaptic NMDARs, similar to that observed with oxA/hcrt-1 (Borgland et al., 2006), in contrast to the presynaptic enhancement of glutamate release, which would likely affect all NMDAR subtypes.

In contrast to the effects of oxA/hcrt-1-mediated potentiation of NMDARs via both OX2/Hcrt-2 and OX1/Hcrt-1 receptors, oxB/hcrt2-mediated potentiation of NMDARs was likely mediated solely by activation of $\mathrm{OX} 2 / \mathrm{Hcrt}-2$ receptors because (1) the OX $1 / \mathrm{Hcrt}-1$ receptor antagonist SB 334867 did not reduce oxB/hcrt-2 potentiation of NMDARs or AMPARs; (2) the putative OX2/Hcrt-2 receptor antagonist JNJ-10397049 blocked potentiation of NMDARs mediated by $10 \mathrm{nM} \mathrm{oxB} /$ hcrt-2, a concentration of $\mathrm{oxB} / \mathrm{hcrt}-2$ that potentiates NMDARs but not AMPARs; and (3) the selective OX2/Hcrt-2 receptor agonist $\mathrm{Ala}^{11}$-D-Leu ${ }^{15}$-orexin B potentiated NMDAR eEPSCs; this is consistent with previous reports that both $\mathrm{OX} 1 / \mathrm{Hcrt}-1$ and OX2/Hcrt-2 receptors are present on VTA dopamine neurons (Korotkova et al., 2003; Narita et al., 2006). OX1/Hcrt-1 and OX2/Hcrt-2 receptors have been proposed to play distinct roles within the central nervous system, in that activation of $\mathrm{OX} 2 / \mathrm{Hcrt}-2$ receptors exhibited a more pronounced functional effect in serotonergic neurons and OX1/Hcrt-1 exhibited a more pronounced functional effect in noradrenergic neurons (Soffin et al., 2004). Additionally, OX2/Hcrt-2 receptor gene mutation or deletion appears to be the primary cause of narcolepsy in dogs or mice, respectively (Chemelli et al., 1999; Lin et al., 1999), and selective stimulation of OX2/Hcrt-2 receptors promotes wakefulness (Akanmu \& Honda, 2005). Therefore, oxB/hcrt-2 enhancement of glutamatergic synaptic transmission via OX2/Hcrt-2 receptors in the VTA may underlie arousal-related effects.

\section{oxB/hcrt-2 inhibits LTP in the VTA}

An enhanced AMPAR/NMDAR ratio in VTA dopamine neurons has been associated with the occlusion of LTP, suggesting that excitatory synapses in the VTA are saturated and no further plasticity can be induced (Ungless et al., 2001). Therefore, the size of induced LTP could be inversely related to the degree to which AMPARs can be trafficked or stabilized at the synapse by LTP stimulation paradigms. For example, if there is an increased AMPAR/NMDAR ratio due to enhanced AMPAR-mediated synaptic transmission, there would be less LTP induced as the synapses are already saturated with AMPARs (Ungless et al., 2001). Previously, we found that oxA/hcrt-1 can potentiate the AMPAR/NMDAR ratio when measured hours after application (Borgland et al., 2006).
Spike-timing-dependent LTP pairs a preceding presynaptic spike with a postsynaptic depolarization (Dan \& Poo, 2004). In the VTA, this plasticity is NMDAR-dependent (Liu et al., 2005). NMDAR activation is thought to be a coincidence detector and primary source of calcium for inducing spike-timing-dependent LTD (Dan \& Poo, 2004). We observed that after application of oxB/hcrt-2 or $\mathrm{Ala}^{11}$ D-Leu ${ }^{15}$-orexin B, LTP induced by spike pairing was less than in untreated cells. OX2/Hcrt-2 receptor activation could initiate a signaling pathway that results in increased intracellular calcium influx through NMDAR activation or other mechanisms (Narita et al., 2007) that facilitate AMPAR trafficking. Once this pathway is engaged, the stimulation protocol for spike-timing-dependent LTP is less effective at trafficking AMPARs to the synapse due to the already activated signal transduction pathway or because the synapses are already partially saturated with AMPARs. Presumably, if we induced LTP at a much later time point after application of oxB/hcrt-2, synapses would be completely saturated and we would be unable to induce LTP. Further experiments are required to determine the exact mechanism of oxB/hcrt-2-mediated reduction in LTP at dopamine neuron synapses.

In conclusion, we have observed that oxB/hcrt-2 caused both preand post-synaptic strengthening of excitatory synaptic transmission in the VTA. Both pre- and post-synaptic effects were mediated by activation of PKC. A recent study suggested that microinjection of a PKC inhibitor directly in the VTA significantly decreased time spent in a context associated with preference to morphine (Narita et al., 2007). Furthermore, the PKC inhibitor blocked an intra-VTA $\mathrm{oxB} /$ hcrt-2-mediated increase in dopamine release in the nucleus accumbens (Narita et al., 2007). Therefore, our results demonstrating a PKC-dependent increase in pre- and post-synaptic excitatory synaptic transmission by oxB/hcrt-2 in the VTA may reflect a strengthening of the association between drugs of abuse and the context in which they are taken.

\section{Acknowledgements}

We thank Drs G. Hjelmstad, F. W. Hopf and R. C. Malenka for helpful comments on the manuscript. We also thank Johnson \& Johnson Pharmaceutical Research \& Development, L.L.C. for its supply of JNJ-10397049. This work was supported by the State of California for medical research on alcohol and substance misuse through the University of California, San Francisco and the National Institutes of Health 1RO1DA15096-01. S.L.B. is supported by an NARSAD young investigators grant.

\section{Abbreviations}

bis, bisindolylmaleimide; EPSC, excitatory post-synaptic current; EPSP, excitatory post-synaptic potential; LTP, long-term potentiation; NMDAR, NMDA receptor; OX1/hrct-1, orexin/hypocretin receptor 1 ; OX2/hrct-2, orexin/hypocretin receptor 2 ; oxA/hrct-1, orexin A/hypocretin 1 ; oxB/hrct2, orexin B/hypocretin 2; PKA, protein kinase A; PKC, protein kinase C; PLC, phospholipase $\mathrm{C}$; TH, tyrosine hydroxylase; VTA, ventral tegmental area.

\section{References}

Akanmu, M.A. \& Honda, K. (2005) Selective stimulation of orexin receptor type 2 promotes wakefulness in freely behaving rats. Brain Res., 1048, 138145.

Balcita-Pedicino, J.J. \& Sesack, S.R. (2007) Orexin axons in the rat ventral tegmental area synapse infrequently onto dopamine and gamma-aminobutyric acid neurons. J. Comp. Neurol., 503, 668-685.

Baldo, B.A., Daniel, R.A., Berridge, C.W. \& Kelley, A.E. (2003) Overlapping distributions of orexin/hypocretin- and dopamine- $\beta$-hydroxylase immuno- 
reactivity fibers in rat brain regions mediating arousal, motivation, and stress. J. Comp. Neurol., 464, 220-237.

Berridge, K.C. (2007) The debate over dopamine's role in reward: the case for incentive salience. Psychopharmacology, 191, 391-431.

Bonci, A. \& Malenka, R.C. (1999) Properties and plasticity of excitatory synapses on dopaminergic and GABAergic cells in the ventral tegmental area. J. Neurosci., 19, 3723-3730.

Borgland, S.L., Malenka, R. \& Bonci, A. (2004) Acute and chronic cocaine-induced potentiation of synaptic strength in the VTA: electrophysiological and behavioral correlates in individual rats. J. Neurosci., 24, 7482-7490.

Borgland, S.L., Taha, S.A., Sarti, F., Fields, H.L. \& Bonci, A. (2006) Orexin A in the VTA is critical for the induction of synaptic plasticity and behavioral sensitization to cocaine. Neuron, 49, 589-601.

Boutrel, B., Kenny, P.J., Specio, S.E., Martin-Fadon, R., Markou, A., Koob, G.F. \& De Lecea, L. (2005) Role for hypocretin in mediating stress-induced reinstatement of cocaine-seeking behavior. Proc. Natl Acad. Sci. USA, 102, $19168-19173$.

Burlet, S., Tyler, C.J. \& Leonard, C.S. (2002) Direct and indirect excitation of laterodorsal tegmental neurons by hypocretin/orexin peptides: implications for wakefulness and narcolepsy. J. Neurosci., 22, 2862-2872.

Cameron, D.L., Wessendorf, M.W. \& Williams, J.T. (1997) A subset of ventral tegmental area neurons is inhibited by dopamine, 5-hydroxytryptamine and opioids. Neuroscience, 77, 155-166.

Carlezon, W. \& Nestler, E.J. (2002) Elevated levels of GLuR1 in the midbrain: a trigger for sensitization to drugs of abuse? Trends Neurosci., 25, 610-615.

Chemelli, R.M., Willie, J.T., Sinton, C.M., Elmquist, J.K., Scammell, T., Lee, C., Richardson, J.A., Williams, S.C., Xiong, Y., Kisanuki, Y., Fitch, T.E., Nakazato, M., Hammer, R.E., Saper, C.B. \& Yanagisawa, M. (1999) Narcolepsy in orexin knockout mice: molecular genetics of sleep regulation. Cell, 98, 437-451.

Dan, Y. \& Poo, M. (2004) Spike timing dependent plasticity of neural circuits. Neuron, 44, 23-30

Date, Y., Mondal, M.S., Matsukura, S. \& Nakazato, M. (2000a) Distribution of orexin-A and orexin-B (hypocretins) in the rat spinal cord. Neurosci. Lett., 288, 87-90.

Date, Y., Mondal, M.S., Matsukura, S., Ueta, Y., Yamashita, H., Kaiya, H., Kangawa, K. \& Nakazato, M. (2000b) Distribution of orexin/hypocretin in rat median eminence and pituitary. Brain Res. Mol. Brain Res., 76, $1-6$.

DeLecea, L., Kilduff, T.S., Peyron, C., Gao, X-B., Foye, P.E., Danielson, P.E., Fukuhara, C., Battenberg, E.L., Gautvik, V.T., Bartless, F.S., Frankel, W.N., van den Pol, A.N., Bloom, F.E., Gautvik, K.M. \& Sutcliffe, J.G. (1998) The hypocretins: hypothalamus-specific peptides with neuroexcitatory activity. Proc. Natl Acad. Sci. USA, 95, 322-327.

Dunn, J.M., Inderwies, B.R., Licata, L.C. \& Pierce, R.C. (2005) Repeated administration of AMPA or a metabotropic glutamate receptor agonist into the rat ventral tegmental area augments the subsequent behavioral hyperactivity induced by cocaine. Psychopharmacology, 179, 172180.

Fadel, J. \& Deutch, A.Y. (2002) Anatomical substrates of orexin-dopamine interactions: lateral hypothalamic projections to the ventral tegmental area. Neuroscience, 111, 379-387.

Fitzgerald, L.W., Ortiz, J., Hamedani, A.G. \& Nestler, E.J. (1996) Drugs of abuse and stress increase the expression of GluR1 and NMDAR1 glutamate receptor subunits in the rat ventral tegmental area: common adaptations among cross-sensitizing agents. J. Neurosci., 16, 274-282.

Ford, C.P., Mark, G.P. \& Williams, J.T. (2006) Properties and opioid inhibition of mesolimbic dopamine neurons vary according to target location. J. Neurosci., 26, 2788-2797.

Georgescu, D., Zachariou, V., Barrot, M., Mieda, M., Willie, J.T., Eisch, A.J., Yanagisawa, M., Nestler, E.J. \& DiLeone, R.J. (2003) Involvement of lateral hypothalamic peptide orexin in morphine dependence and withdrawal. J. Neurosci., 23, 3106-3111.

Grace, A.A. \& Onn, S.P. (1989) Morphology and electrophysiological properties of immunocytochemically identified rat dopamine neurons recorded in vitro. J. Neurosci., 9, 3463-3481.

Grace, A.A., Gerfern, C.R. \& Aston-Jones, G. (1998) Catecholamines in the central nervous system. Adv. Pharmacol., 42, 655-670.

Harris, G.C. \& Aston-Jones, G. (2006) Arousal and reward: a dichotomy in orexin function. Trends Neurosci., 29, 571-577.

Harris, G.C., Wimmer, W. \& Aston-Jones, G. (2005) A role for lateral hypothalamic orexin neurons in reward seeking. Nature, 437, 556559.
Johnson, S.W. \& North, R.A. (1992) Two types of neurone in the rat ventral tegmental area and their synaptic inputs. J. Physiol., 450, 455468.

Kastin, A.J. \& Akerstrom, V. (1999) Orexin A but not orexin B rapidly enters brain from blood by simple diffusion. J. Pharmacol. Exp. Ther., 289, 219223

Kauer, J.A. (2004) Learning mechanisms in addiction: synaptic plasticity in the ventral tegmental area as a result of exposure to drugs of abuse. Annu. Rev. Physiol., 66, 447-475

Korotkova, T.M, Sergeeva, O.A., Eriksson, K.S., Haas, H.L. \& Brown, R.E. (2003) Excitation of ventral tegmental area dopaminergic and nondopaminergic neurons by orexin/hypocretins. J. Neurosci., 23, 7-11.

Lacey, M.G., Mercuri, N.B. \& North, R.A. (1990) Actions of cocaine on rat dopaminergic neurons in vitro. Br. J. Pharmacol., 99, 731-735.

Lawrence, A.J., Cowen, M.S., Yang, H-J, Chen, F. \& Oldfield, B. (2006) The orexin system regulates alcohol-seeking in rats. Br. J. Pharmacol., 148, 752 759

Lin, L., Faraco, J., Li, R., Kadotani, H., Rogers, W., Lin, X., Qiu, X., de Jong, P.J., Nishino, S. \& Mignot, E. (1999) The sleep disorder canine narcolepsy is caused by a mutation in the hypocretin (orexin) receptor 2 gene. Cell, 98 , 365-376.

Liu, Q.S., Pu, L. \& Poo, M.M. (2005) Repeated cocaine exposure in vivo facilitates LTP induction in midbrain dopamine neurons. Nature, 437, 1027 1031.

Malenka, R.C., Madison, D.V. \& Nicoll, R.A. (1986) Potentiation of synaptic transmission in the hippocampus by phorbol esters. Nature, 321, $175-177$.

Margolis, E.B., Lock, H., Hjelmstad, G.O. \& Fields, H.L. (2007) The ventral tegmental area revisited: is there an electrophysiological marker for dopaminergic neurons? J. Physiol., 577, 904-924.

McAtee, L.C., Sutton, S.W., Rudolph, D.A., Li, X., Aluisio, L.E., Phuong, V.K., Dvorak, C.A., Lovenberg, T.W., Carruthers, N.I. \& Jones, T.K.. (2004) Novel substituted 4-phenyl-[1,3]dioxanes: potent and selective orexin receptor $2(\mathrm{OX}(2) \mathrm{R})$ antagonists. Bioorg. Med. Chem. Lett., 14 4225-4229.

Mondal, M.S., Nakazato, M., Date, Y., Murakami, N., Hanada, R., Sakata, T. \& Matsukura, S. (1999a) Characterization of orexin-A and orexin-B in the microdissected rat brain nuclei and their contents in two obsess rat models. Neursoc. Lett., 273, 45-48.

Mondal, M.S., Nakazato, M., Date, Y., Murakami, N., Yanigasawa, M. \& Matsukura, S. (1999b) Widespread distribution of orexin in rat brain and its regulation upon fasting. Biochem. Biophys. Res. Commun., 25, 495599

Narita, M., Nagumo, Y., Hashimoto, S., Narita, M., Khotib, J., Miyatake, M., Sakurai, T., Yanagisawa, M., Nakamachi, T., Shioda, S. \& Suzuki, T. (2006) Direct involvement of orexinergic systems in the activation of the mesolimbic dopamine pathway and related behaviors induced by morphine. J. Neurosci., 26, 398-405.

Narita, M., Nagumo, Y., Miyatake, M., Ikegami, D., Kurahashi, K. \& Suzuki, T. (2007) Implication of protein kinase $C$ in the orexin-induced elevation of extracellular dopamine levels and its rewarding effect. Eur. J. Neurosci., 25, 1537-1545.

Overton, P.G., Richards, C.D., Berry, M.S. \& Clark, D. (1999) Long-term potentiation at excitatory amino acid synapses on midbrain dopamine neurons. Neuroreport, 10, 221-226.

Peyron, C., Tighe, D.K., van den Pol, A., de Lecea, L., Heller, H.C., Sutcliffe, J.G. \& Kilduff, T.S. (1998) Neurons containing hypocretin (orexin) project to multiple neuronal systems. J. Neurosci., 18, 9996-10015.

Saal, D., Dong, Y., Bonci, A. \& Malenka, R.C. (2003) Drugs of abuse and stress trigger a common synaptic adaptation in dopamine neurons. Neuron, 37, 577-582.

Sakurai, T., Amemiya, A., Ishii, M., Matsuzaki, I., Chemelli, R.M., Tanaka, H., Williams, S.C., Richardson, J.A., Kozlowski, G.P., Wilson, S., Arch, J.R. Buckingham, R.E., Haynes, A.A.C., Carr, S.A., Annan, R.S., McNulty, D.E., Liu, W.S., Terrett, J.A., Elshourbagy, N.A., Bergsma, D.J. \& Yanagisawa, M. (1998) Orexin and orexin receptors: a family of hypothalamic neuropeptides and $\mathrm{G}$ protein-coupled receptors that regulate feeding behavior. Cell, 92, $573-585$.

Soffin, E.M., Gill, C.H., Brough, S.J., Jerman, J.C. \& Davies, C.H. (2004) Pharmacological characterization of the orexin receptor subtype mediating postsynaptic excitation in the rat dorsal raphe nucleus. Neuropharmacology, 46, 1168-1176.

Terrian, D.M., Ways, D.K. \& Gannon, R.L. (1991) A presynaptic role for protein kinase $\mathrm{C}$ in hippocampal mossy fiber synaptic transmission. Hippocampus, 1, 303-314. 
1556 S. L. Borgland et al.

Ungless, M.A., Whistler, J.L., Malenka, R.C. \& Bonci, A. (2001) A single cocaine exposure in vivo induces long-term potentiation in dopamine neurons. Nature, 411, 583-587.

Uramura, K., Funahashi, H., Muroya, S., Shioda, S., Takigawa, M. \& Yada, T. (2001) Orexin A activates phospholipase C- and protein kinase C-mediated $\mathrm{Ca}^{2+}$ signaling in dopamine neurons of the ventral tegmental area Neuroreport, 12, 1885-1889.

Van den pol, A.N., Gao, X.B., Obrietan, K., Kilduff, T.S. \& Belousov, A.B. (1998) Presynaptic and postsynaptic actions and modulation of neuroendocrine neurons by a new hypothalamic peptide, hypocretin/orexin. J. Neurosci., 18, 7962-7971.
Wise, R. (1996) Addictive drugs and brain stimulation reward. Annu. Rev. Neurosci., 19, 319-340.

Wolf, M.E., Sun, X., Mangiavacchi, S. \& Chao, S.Z. (2004) Psychomotor stimulants and neuronal plasticity. Neuropharmacology, 49, 61-79.

Yamanaka, A., Beuckmann, C.T., Willie, J.T., Hara, J., Tsujino, N., Mieda, M., Tomnaga, M., Yagami, K., Sugiyama, F., Goto, K., Yanagisawa, M. \& Sakurai, T. (2003) Hypothalamic orexin neurons regulate arousal according to energy balance in mice. Neuron, 38, 701-713.

Zucker, R.S. \& Regehr, W.G. (2002) Short term plasticity. Ann. Rev. Physiol., 64, 355-405. 